\title{
Protease-Activated Receptors - Key Regulators of Inflammatory Bowel Diseases Progression
}

\author{
Damian Jacenik (1) \\ Jakub Fichna ${ }^{2}$ \\ Ewa Małecka-Wojciesko ${ }^{3}$ \\ Anna Mokrowiecka ${ }^{3}$ \\ 'Department of Cytobiochemistry, \\ Faculty of Biology and Environmental \\ Protection, University of Lodz, Lodz, \\ Poland; ${ }^{2}$ Department of Biochemistry, \\ Faculty of Medicine, Medical University of \\ Lodz, Lodz, Poland; ${ }^{3}$ Department of \\ Digestive Tract Diseases, Faculty of \\ Medicine, Medical University of Lodz, \\ Lodz, Poland
}

Correspondence: Damian Jacenik Department of Cytobiochemistry, Faculty of Biology and Environmental Protection, University of Lodz, 141// 43 Pomorska St. 90-236 Lodz, Poland

Tel +4842635 5299

Fax +48426354484

Email damian.jacenik@biol.uni.lodz.pl

\begin{abstract}
The pathogenesis and course of inflammatory bowel diseases are related to both immune system disorders and dysfunction of colon permeability. Moreover, co-existing diseases in patients with Crohn's disease and ulcerative colitis are identified. Currently, there are some therapeutic strategies that affect the function of cytokine/s causing inflammation in the intestinal wall. However, additional approaches which target other components of inflammatory bowel diseases pathogenesis are still needed. Accumulating evidence suggests that proteases and protease-activated receptors seem to be responsible for colitis progression. Experimental and observational studies showed alteration of protease-activated receptors expression in the colon of patients with Crohn's disease and ulcerative colitis. Furthermore, it was suggested that the expression of protease-activated receptors correlated with inflammatory bowel diseases activity. Moreover, regulation of protease-activated receptors seems to be responsible for the modulation of colitis and clinical manifestation of inflammatory bowel diseases. In this review, we present the current state of knowledge about the contribution of protease-activated receptors to Crohn's disease and ulcerative colitis and its implications for diagnosis and treatment.
\end{abstract}

Keywords: inflammatory bowel disease, Crohn's disease, ulcerative colitis, colitis, immune response, proteinase-activated receptor

\section{Introduction}

Deregulated immune response plays a major role in the pathogenesis of inflammatory bowel diseases (IBD). However, still little is known about the processes related to immune response which support immune cells infiltration and loss of cell-cell interaction. These phenomena allow expansion of immune cells across inflamed tissues and induce production of cytokines and chemokines. In fact, communication between intestinal epithelial cells and immune cells plays a crucial role in the modulation of epithelial cell function affecting junctional complexes between inflammatory cells and intestinal epithelial cells that might contribute to the dysregulation of intestinal epithelial barrier. ${ }^{1}$ Accumulating evidence suggests that those proteases seem to be crucial enzymes responsible for the above-mentioned processes and are able to stimulate progression of colitis and affect therapy efficiency. Mammalian proteases are classified as serine, threonine, cysteine and aspartic proteases as well as metalloproteases (known as metalloproteinases - MMPs) and are widely distributed in the human body, including in the gastrointestinal tract. ${ }^{2}$ In IBD patients, disturbed expression of proteases has been reported by numerous studies. For instance, Curciarello et al. documented that the colon of patients with colitis is manifested by higher activity of neutrophils' elastase. ${ }^{3}$ In line, enhanced 
expression of numerous MMPs in the colon of patients with IBD was documented and the expression of MMPs seems to be a predictor/prognostic factor in IBD. ${ }^{4-7}$ The action of MMP affects the efficiency of biological therapy in patients with IBD. ${ }^{8}$ Therapy against tumor necrosis factor- $\alpha$ (TNF- $\alpha)$ or anti- $\alpha 4$-integrin is now the most effective approach for IBD patients. ${ }^{9}$ However, accumulating findings indicate that about $40 \%$ of patients with IBD have been identified as non-responding to treatment and many of the patients treated with anti-TNF- $\alpha$ agents are developing resistance to the therapy. ${ }^{10,11}$ Biancheri et al. found that both MMP3 and MMP12 are responsible for the degradation of TNF- $\alpha$ agents, i.e. infliximab, adalimumab and etanercept, which is directly related to the loss of their ability to neutralize TNF- $\alpha{ }^{8}$ As shown by Curciarello et al., neutrophils' elastase cleaved biological agents leading to loss of function monoclonal antibodies and abovementioned findings may explain the phenomenon of the non-responsiveness to the treatment in the IBD patients. ${ }^{3}$

Although proteases are present in the gastrointestinal tract per se, they can also be released by microbiota. Consequently, the fact that the gastrointestinal tract is exposed to various serine proteases was confirmed by studies where protease content in the colon and fecal matter of IBD patients was analyzed. ${ }^{12-15}$ It was documented that Crohn's disease (CD) and ulcerative colitis (UC) patients are characterized by 10- and 9-fold higher protease activity in fecal matter compared to healthy subjects. ${ }^{12}$ As previously described by Galipeau et al., the proteolytic signature in fecal matter in asymptomatic individuals at risk for IBD may be a non-invasive marker of inflammation. ${ }^{16,17}$ On the other hand, modification of microbiota composition seems to be a promising supportive approach in the therapy of IBD patients and may reduce protease activity. Nevertheless, the fecal microbiota transplant procedure, including material preparation, donor/s selection and microbiota profiling, resulting in proteolytic activity inhibition must be optimized and further investigated in pre-clinical and clinical studies.

The four members of the protease-activated receptors (PARs) family, i.e. $\mathrm{PAR}_{1}, \mathrm{PAR}_{2}, \mathrm{PAR}_{3}$, and $\mathrm{PAR}_{4}$, were identified and are encoded by the $F 2 R, F 2 R L 1, F 2 R L 2$ and $F 2 R L 3$ gene, respectively. The mechanism of PARs activation is associated with the proteases action that cleaves a specific site in the extracellular amino terminus of the PARs, which then generates a new extracellular amino terminus serving as a tethered ligand domain. ${ }^{18,19}$ The tethered ligand domain binds to a highly conserved region in the second extracellular loop of the cleaved PAR; additionally, exposed tethered ligand domain of certain PARs can bind and activate other PARs. ${ }^{20,21}$ PARs activation affects $G$ protein-dependent signal transduction, involving several types of $\mathrm{G}$ protein $\alpha$, i.e. $\mathrm{G} \alpha_{\mathrm{q}}, \mathrm{G} \alpha_{12 / 13}, \mathrm{G} \alpha_{\mathrm{i}}$ and $\mathrm{G} \beta \gamma$, as well as $\beta$-arrestin. ${ }^{22-25}$

Accumulating observational and experimental evidence indicates that activity modulation of PARs may affect numerous aspects in the progression of IBD. In this review, we present and elaborate on current knowledge concerning the role of PARs in processes engaged in immune response and colon permeability during colitis progression as well as the clinical significance of PARs expression and signaling in $\mathrm{CD}$ and $\mathrm{UC}$.

\section{Expression of Protease-Activated Receptors in Inflammatory Bowel Diseases}

A growing body of studies has documented that $\mathrm{PAR}_{1}$, $\mathrm{PAR}_{2}$ and $\mathrm{PAR}_{4}$ are expressed differently in the colon of patients with $\mathrm{CD}$ and $\mathrm{UC}$ in comparison to healthy controls. Moreover, deregulated expression of PARs in animal models of colitis has also been observed. ${ }^{26-29}$

For instance, it was proven that $\mathrm{PAR}_{1}$ is overexpressed in the inflamed colon obtained from $\mathrm{CD}$ patients in comparison to the non-inflamed colon. Saeed et al. documented that $\mathrm{PAR}_{1}$ is overexpressed in the colon of pediatric patients characterized by severe stage of CD compared to healthy subjects and pediatric patients with moderate stage of $\mathrm{CD}^{29}$ In line, $\mathrm{PAR}_{2}$ is widely expressed in the healthy human colon and is localized in both villi and crypts of the epithelial cells. $^{30,31}$

Immunohistochemistry analysis performed by Kim et al. and $\mathrm{Ke}$ et al. documented that $\mathrm{PAR}_{2}$ is overexpressed in lamina propria of CD and UC patients when compared to healthy subjects. ${ }^{32,33}$ Interestingly, higher expression of $\mathrm{PAR}_{2}$ at the mRNA and protein level was also documented in duodenal mucosa and epithelial cells obtained from dogs with IBD when compared to healthy subjects. ${ }^{31}$ In line, higher expression of $\mathrm{PAR}_{2}$ in the colon epithelium of CD patients, but not UC patients, in comparison to healthy subjects has been shown. ${ }^{33}$ However, epigenetic regulation of F2RL1 gene promoter in the colon of UC patients may be the reason why, in some studies, lack of changes in the expression of $\mathrm{PAR}_{2}$ between UC patients and healthy subjects was documented. Indeed, Tahara et al. and Gould et al. found enhanced methylation status of F2RL1 
gene promoter in the colon of patients with UC characterized by more extensive colitis and steroid-dependent type as well as refractory type of UC. ${ }^{34,35}$

Finally, $\mathrm{PAR}_{4}$ is expressed in the colon but the significance of $\mathrm{PAR}_{3}$ and $\mathrm{PAR}_{4}$ expression and action mediated by both receptors in the gastrointestinal tract remains elusive. ${ }^{26,28}$ However, Dabek et al. found that the expression of $\mathrm{PAR}_{4}$ is higher in the colon of UC patients compared to healthy subjects. ${ }^{28}$

\section{Protease-Activated Receptors Modulate Immune Response in Inflammatory Bowel Diseases}

Accumulating evidence demonstrates that $\mathrm{PAR}_{1}$ and $\mathrm{PAR}_{2}$ are involved in the modulation of immune response in IBD. ${ }^{13,29-32,36-41}$ Immunomodulatory action of $\mathrm{PAR}_{1}$ in the colon was estimated by Cenac et al., who used specific peptides which act as $\mathrm{PAR}_{1}$ agonists. ${ }^{37}$ In an animal model of oxazolone-induced colitis, selective activation of $\mathrm{PAR}_{1}$ reduced macroscopic colon damage score and myeloperoxidase activity as well as colon wall thickness in comparison to mice treated with oxazolone and control peptide. To confirm the effects of $\mathrm{PAR}_{1}$ activation on colitis regulation, the impact of $\mathrm{PAR}_{1}$ peptide ligands in oxazolone-treated wild type mice and mice with deletion of $F 2 r$ gene was also evaluated. In fact, both approaches indicated that $\mathrm{PAR}_{1}$ affected colitis severity in in vivo models.

It also has to be mentioned that modulation of $\mathrm{PAR}_{1}$ activity regulates the expression of cytokines in the inflamed colon. The link between $\mathrm{PAR}_{1}$ and proinflammatory cytokines was revealed by Saeed et al., who found that changes in the expression of $\mathrm{PAR}_{1}$ in the colon of patients with IBD correlate with changes in the expression of several cytokines. ${ }^{29} \mathrm{PAR}_{1}$ expression is positively correlated with the expression of numerous interleukins (ILs), such as $I L 17$ and IL23A, which was documented in the colon of pediatric $\mathrm{CD}$ patients with severe colitis. Moreover, it was suggested that $\mathrm{PAR}_{1}$ may participate in the regulation of immune response mediated by IL-17 producing T cells, i.e. Th17 (Figure 1). Of note, the mechanism responsible for naïve $\mathrm{T}$ cell differentiation to Th17 cells associated with $\mathrm{PAR}_{1}$ action seems to be mediated by macrophages. An ex vitro approach, whereby Saeed et al. used peritoneal macrophages and inflammatory stimuli, revealed that wild type and $F 2 R^{-/-}$macrophages are able to produce pro-inflammatory cytokines such as IL-6 but only wild type and not $F 2 R^{-/-}$ macrophages produced IL-23. ${ }^{29}$ It suggests that $\mathrm{PAR}_{1}$ is the essential PAR responsible for IL-23 production in activated macrophages. It should be noted that $\mathrm{T}$ cells activated by IL-23 are able to promote colitis and this phenomenon is mediated by both IL-6 and IL-17, which has been proven using an in vivo approach. ${ }^{42}$ Abovementioned observations strongly suggest that $\mathrm{PAR}_{1}$ regulates IL-23 production in the response of inflammatory stimuli which may affect naïve $\mathrm{T}$ cell polarization to Th17 cells in the colon of IBD patients. In line, Saeed et al found a similar expression profile of genes (IL17, $I L 22$ and $I L 23 A$ ) related to Th17 cells in the colon of Citrobacter rodentium-induced model of colitis in wild type mice but not in animals with $\mathrm{PAR}_{1}$ knockout. $^{29}$ On the other hand, lower production of several cytokines such as IL-6, IL-17, IL-23, and interferon (IFN)- $\gamma$ in splenocytes of mice with $\mathrm{PAR}_{1}$ knockout compared to wild type splenocytes was noted. Collectively, in vivo studies found that $\mathrm{PAR}_{1}$ seems to be capable of modulating both systemic and local immune response mediated by Th1 and Th17, respectively. Collectively, pre-clinical studies indicate that $\mathrm{PAR}_{1}$ antagonists can be potent agents blocking not only specific cytokine/s production but seem to be responsible for more complex action of immune cells.

Studies taking into consideration PARs activity highlighted functional significance of $\mathrm{PAR}_{2}$ action in several aspects of immune response in IBD. Patel et al. documented that administration of a major serine protease which acts as $\mathrm{PAR}_{2}$ agonist, i.e. trypsin, is responsible for development of more severe colitis when compared to untreated animals with induced colitis. ${ }^{39}$ Additionally, it was found that trypsintreated rats with colitis were characterized by enhanced loss of body weight, food and water intake and poorer colonic mucosal damage as well as disease activity index compared to untreated animals with colitis. ${ }^{39}$ It was found that trypsin administration is associated with higher activity of myeloperoxidase and mast cell degranulation and this phenomenon was also enhanced in trypsin-treated animals with colitis compared to untreated rats with colitis. ${ }^{39}$ In another study, increased wall thickness and macroscopic damage score as well as higher activity of granulocyte infiltration was documented in the colons of wild type mice treated with endogenous proteases such as trypsin and tryptase as well as specific peptides that act as $\mathrm{PAR}_{2}$ agonists; moreover, those effects were not observed when inactive enzymes and control peptides were employed. ${ }^{30}$ Additionally, the colons of wild type mice after administration of $\mathrm{PAR}_{2}$ specific peptide were characterized by edema and erosion in the submucosa and 


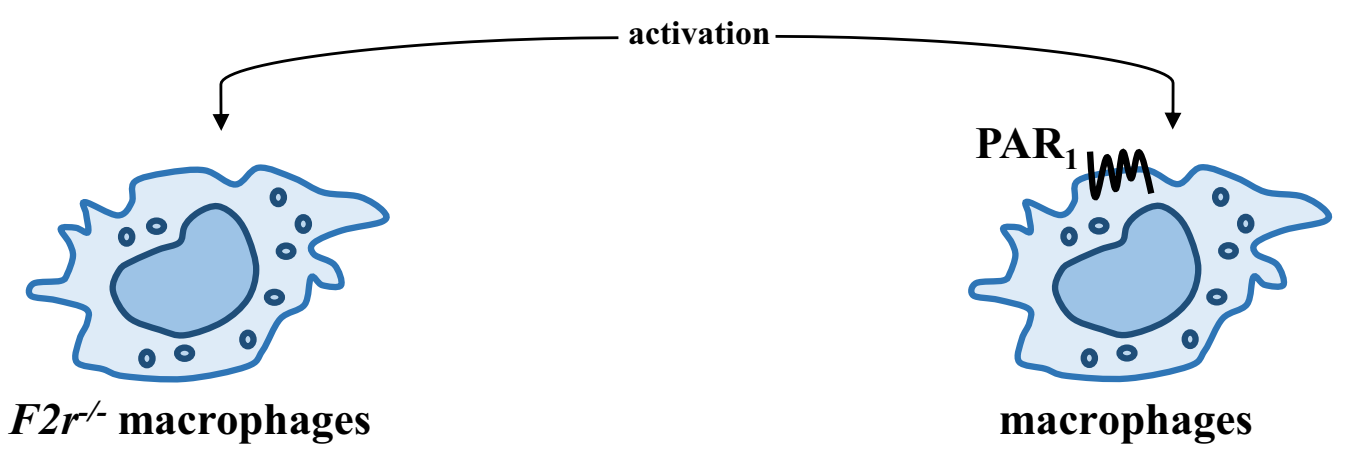

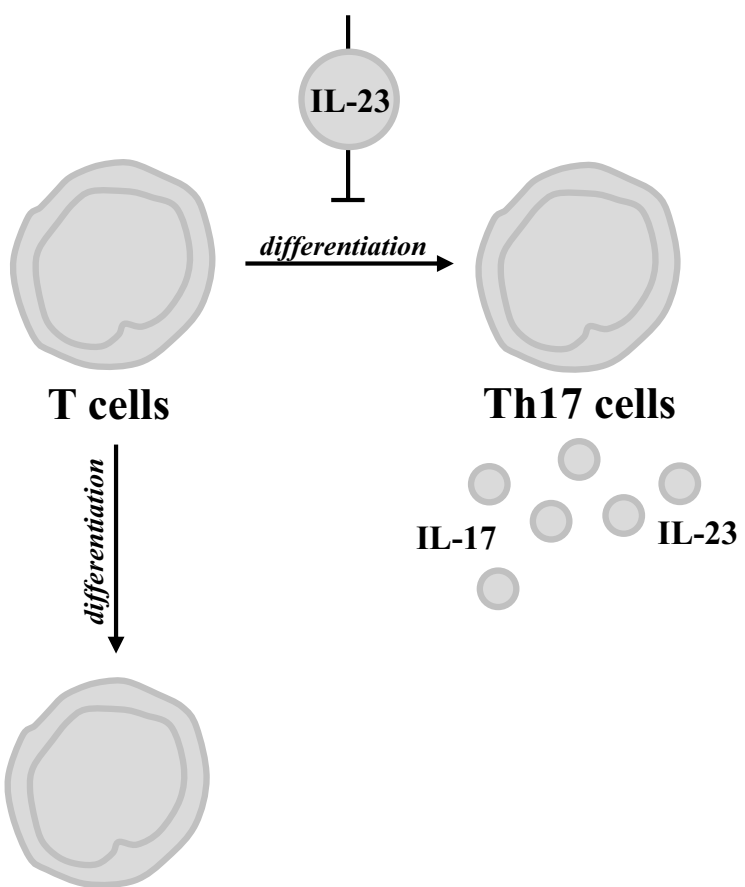

Th1 cells

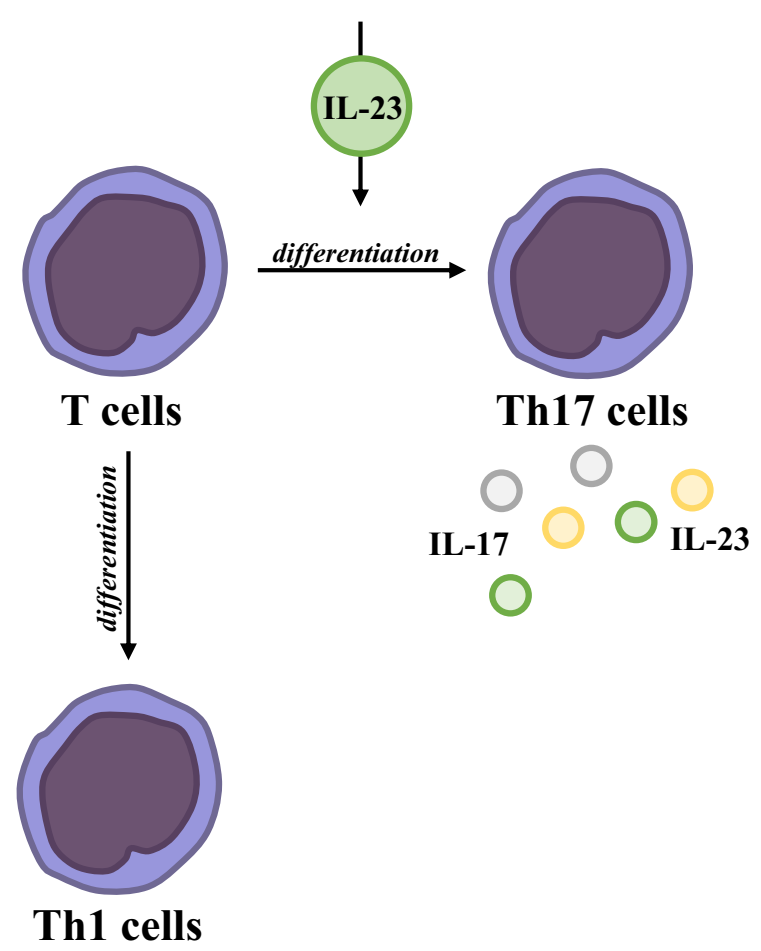

Figure I Impact of PAR I on immune response mediated by macrophages and T cells. PAR I activation affects IL-23 secretion from macrophages, which induces T cell differentiation.

epithelium, respectively. Histological analysis of the colon obtained from F2rll knockout mice treated with $\mathrm{PAR}_{2}$ agonist revealed lack of changes at the microscopic level. ${ }^{30}$ Maeda et al., using an ex vivo approach, documented that treatment of duodenal tissue with trypsin or specific peptide which acts as a specific $\mathrm{PAR}_{2}$ agonist led to enhanced expression of $I l 1 \beta$, Il8 and mucosae-associated epithelial chemokine as well as fractalkine; over-expression of cytokines and chemokines was abolished when trypsin and $\mathrm{PAR}_{2}$ specific peptides in combination with a serine protease inhibitor were employed. $^{31}$ The above-mentioned observations highlighted crucial role of $\mathrm{PAR}_{2}$ in the progression of colitis and documented that $\mathrm{PAR}_{2}$ regulates both cytokines and chemokines expression.
Moreover, $\mathrm{PAR}_{2}$ action affects a wide spectrum of immune and immune-related cells during progression of colitis. For instance, it was proven that $\mathrm{CD}^{+} \mathrm{T}$ cells expressed $\mathrm{PAR}_{2}$ and seem to be directly linked with BCL2like protein 12 (BCL2L12) action to promote Th2-related immune response in patients with UC. ${ }^{40,43}$ Studies conducted by Feng et al., based on human samples, revealed that BCL2L12 expression in $\mathrm{CD}^{+} \mathrm{T}$ cells correlated with both tryptase and Th2-related cytokine levels in serum of UC patients. ${ }^{40}$ Additionally, Feng et al. were able to prove that $\mathrm{PAR}_{2}$ activation by tryptase and/or $\mathrm{PAR}_{2}$ specific peptide affects expression of IL-4, IL-5 as well as IL-13, and knockout of BCL2L12 gene abolishes up-regulation of Th2related cytokine expression and binding of GATA3 
transcription factor to promoter loci of the above-mentioned cytokines in $\mathrm{CD}^{+} \mathrm{T}$ cells. Moreover, it was proven that $\mathrm{PAR}_{2}$ is required for up-regulation and stabilization of BCL2L12 expression, which seems to be crucial for inhibition of spontaneous $\mathrm{CD}^{+}$T cell decays. Finally, Feng et al., using wild type and $B c l 2 l 12^{-/-}$mice, documented that BCL2L12 is needed for induction of Th2-mediated immune response regulated by $\mathrm{PAR}_{2}$ in the colon. ${ }^{40}$

Another study highlighted that the mechanism by which $\mathrm{PAR}_{2}$ affects colitis progression may be directly related to action mediated by mast cells. The colon of patients with IBD and the colons obtained from murine model of colitis are characterized by increased infiltration of mast cells. ${ }^{38,41}$ Christerson et al. found that uninflamed and inflamed colons of patients with $\mathrm{CD}$ are characterized by enhanced number of $\mathrm{PAR}_{2}{ }^{+}$mast cells when compared to colons of healthy subjects. $^{38}$ On the other hand, higher levels of extracellular matrix proteins in both human and mouse colons with colitis were noted, which suggests that mast cell tryptase may be crucial in fibrosis promotion during IBD progression. This hypothesis was confirmed by a study conducted by Liu et al., whereby mast cell deficient mice were used, and similar effects were observed when tryptase inhibitor in wild type mice with colitis was employed. ${ }^{41}$ It also has to be mentioned that both mast cells and tryptase are capable of fibroblast activation and initiation of collagen and fibronectin secretion. ${ }^{41}$ According to Kim et al., over $60 \%$ of tryptase ${ }^{+}$mast cells in the colon of UC patients expressed both $\mathrm{PAR}_{2}$ and tumor necrosis factor (TNF)- $\alpha$, which suggests that there is a cell-specific link between PARs and inflammation. ${ }^{32}$ An in vitro study employing human mast cells revealed that HMC-1 cells treated with trypsin, tryptase or specific $\mathrm{PAR}_{2}$ peptide are capable of $\mathrm{TNF}-\alpha$ secretion, and this effect was not observed when serine protease inhibitor and/or control peptide were used. A bidirectional link between TNF- $\alpha$ and $\mathrm{PAR}_{2}$ was also documented by Christerson et al., who, using HMC-1 cells, were able to note $\mathrm{PAR}_{2}$ overexpression in activated mast cells. ${ }^{38}$ Finally, it was documented by Christerson et al. that TNF- $\alpha$ leads to $\mathrm{PAR}_{2}$ up-regulation in the intestinal myofibroblasts, which are the primary cell type involved in secretion of extracellular matrix during fibrosis. ${ }^{44}$ These findings suggest that $\mathrm{PAR}_{2}$ action is directly associated with colitis and fibrosis promotion (Figure 2). Moreover, $\mathrm{PAR}_{2}$ activation led to the promotion of the growth of intestinal myofibroblasts, which seems to be mediated by cytosolic phospholipase A2. ${ }^{44}$

Most of the evidence noted a positive association between up-regulation and activation of $\mathrm{PAR}_{2}$ with colitis promotion. However, there are also opposite findings which should be mentioned. For instance, Fiorucci et al. found that $\mathrm{PAR}_{2}$ activation by a specific peptide protects the colon from colitis, and that $\mathrm{PAR}_{2}$ activity modulation was responsible for mortality reduction in mice treated with TNBS. ${ }^{36}$ Administration of $\mathrm{PAR}_{2}$ specific peptide, which acts as an agonist, reduced pro-inflammatory cytokine expression in the colon of mice with colitis. The mechanisms responsible for colitis reduction seem to be related to lower content of the $\mathrm{T}$ cells in the colon of mice with colitis treated with $\mathrm{PAR}_{2}$ agonist. It was documented that TNBS administration led to up-regulation of CD44 expression in the $\mathrm{T}$ cells obtained from lamina propria when compared to untreated
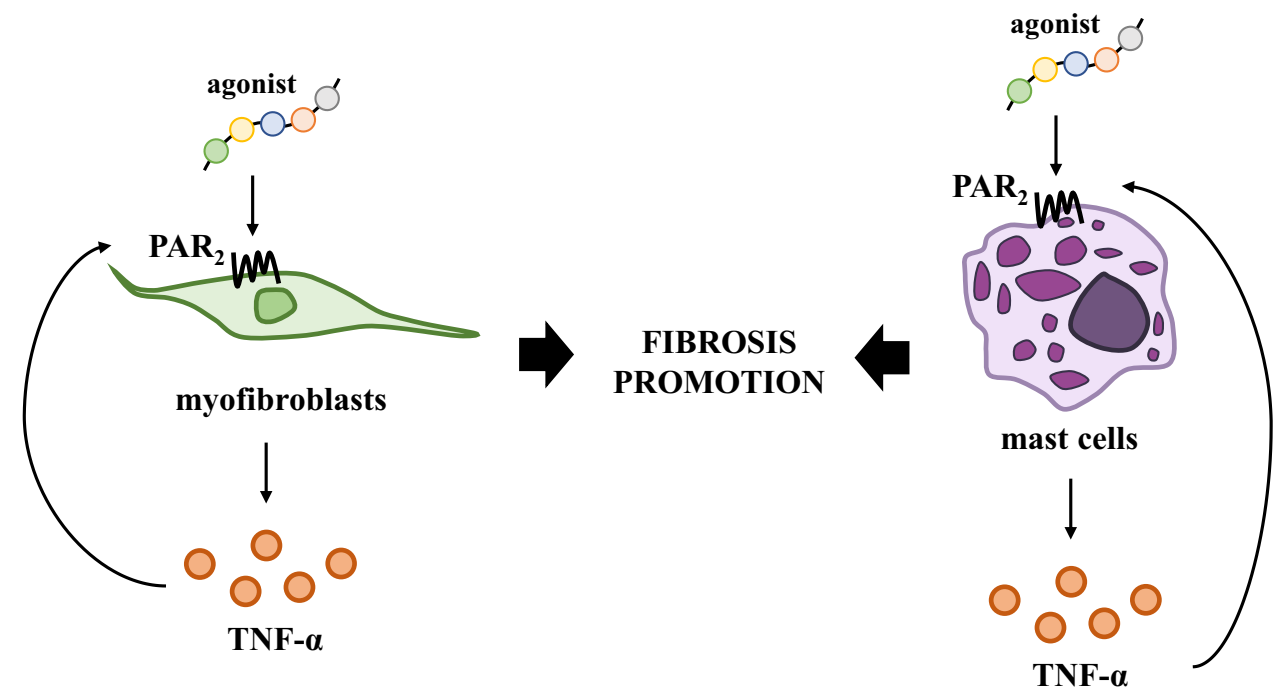

Figure 2 Significance of $\mathrm{PAR}_{2}$ in the promotion of fibrosis mediated by myofibroblasts and mast cells. PAR 2 activation induces TNF- $\alpha$ secretion affecting colitis progression and promotion of fibrosis which is regulated by both myofibroblasts and mast cells. 
animals. Moreover, $\mathrm{PAR}_{2}$ specific peptide was able to reduce proliferation of stimulated $\mathrm{T}$ cells obtained from lamina propria of TNBS-treated mice, which was also associated with lower production of IFN- $\gamma$ in vitro. ${ }^{36}$

Collectively, accumulating results indicate that $\mathrm{PAR}_{1}$ and $\mathrm{PAR}_{2}$ are crucial members of PARs which are involved in the modulation of immune response in IBD. In fact, studies indicated that PARs ligands can be potent agents blocking not only specific cytokine/s production but seem to be responsible for more complex action of immune cells. However, so far only limited efforts have been taken to explore the impact of $\mathrm{PAR}_{4}$ during colitis. Moreover, it also has to be highlighted that conflicting results about $\mathrm{PAR}_{2}$ significance exist and further research is needed to estimate $\mathrm{PAR}_{2}$ action in immune response during colitis.

\section{Protease-Activated Receptors} Affect Colon Permeability and Apoptosis of Colonic Epithelial Cells in Inflammatory Bowel Diseases

PARs - beside immune response modulation, which is the main hallmark of colitis - seem to be involved in the regulation of colon permeability and this phenomenon also affects the action of immune cells in the colon. In fact, the mechanism mediated by $\mathrm{PAR}_{1}$ and $\mathrm{PAR}_{2}$ directly related to colon permeability and neutrophil infiltration across the colon was documented (Figure 3).

Chin et al., using monolayer culture, were able to show that neutrophil and neutrophil's elastase as well as proteinase-3 are responsible for down-regulation of transepithelial resistance. ${ }^{27}$ Additionally, it was evaluated that the regulation of epithelial permeability initiated by both $\mathrm{PAR}_{1}$ and $\mathrm{PAR}_{2}$ activation affects myosin $\mathrm{L}$ chain kinase (MLCK) stimulation and myosin L chain phosphorylation. Of note, MLCK plays a critical role in the regulation of epithelial paracellular permeability and the expression of MLCK may be modulated by pro-inflammatory cytokines, and these phenomena are crucial in maintaining proper tight junction in the colon. ${ }^{45-47}$ It should also be highlighted that both deregulated processes in the colon of patients with IBD, i.e. immune response and colon permeability co-exist and work as a positive feedback loop affecting IBD progression. Chin et al. found that siRNAs against both $\mathrm{PAR}_{1}$ and $\mathrm{PAR}_{2}$ improved transepithelial resistance and decreased transepithelial migration of

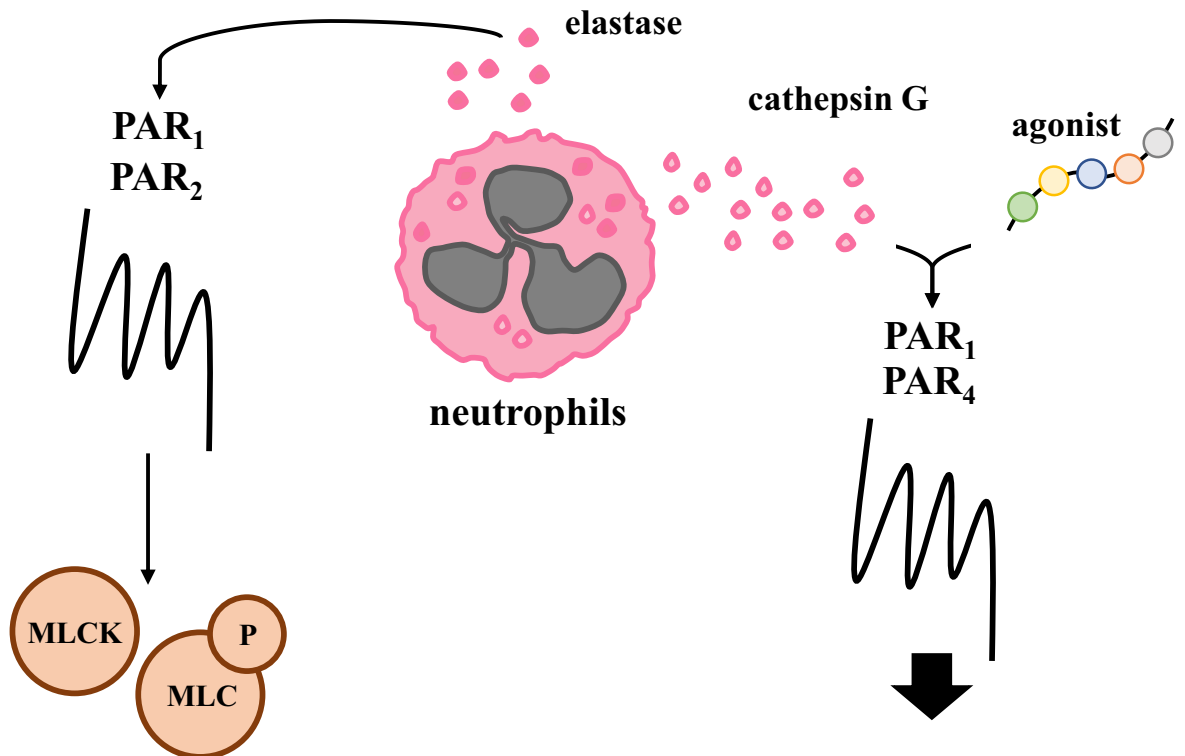

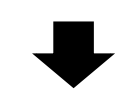

macroscopic damage

granulocyte infiltration in the colon

Figure 3 Experimentally proved processes mediated by PARs during the progression of colitis. PARs agonists and neutrophil proteases modulate granulocyte infiltration by MLCK and MLC axis and affect macroscopic damage and colon permeability in an in vivo model of colitis. 
neutrophils while lack of changes was observed when only one siRNA against $\mathrm{PAR}_{1}$ or $\mathrm{PAR}_{2}$ was employed. ${ }^{27}$ The above-mentioned phenomena prove that cooperation of both PARs is crucial in the regulation of epithelial permeability.

Besides $\mathrm{PAR}_{1}$ and $\mathrm{PAR}_{2}, \mathrm{PAR}_{4}$ also seem to be involved in the regulation of colon permeability in IBD (Figure 3). It was documented that a specific peptide which acts as a $\mathrm{PAR}_{4}$ agonist, cathepsin G, which is a neutrophil granule protease, also acts as an activator of $\mathrm{PAR}_{4}$, and that supernatants obtained from $\mathrm{CD}$ and $\mathrm{UC}$ patients' feces are capable of inducing changes in the colonic paracellular permeability and enhanced activity of myeloperoxidase in the colons of mice with colitis. Moreover, supernatants obtained from feces of UC patients are enriched in serine proteases when compared to healthy subjects and may affect the action of PARs in in vitro and in vivo studies. ${ }^{48,49}$ Interestingly, both $\mathrm{PAR}_{1}$ and/ or $\mathrm{PAR}_{2}$ antagonists administration did not affect changes in colonic paracellular permeability. On the contrary, $\mathrm{PAR}_{4}$ and cathepsin $G$ antagonists decreased the level of myeloperoxidase activity in the colon, which suggests that neutrophil granule protease and $\mathrm{PAR}_{4}$ cooperation may be crucial for proper barrier function and colitis progression in the colon. ${ }^{28,50}$

Apoptosis of colonic epithelial cells is another aspect mediated by PARs which is crucial in maintaining intestinal homeostasis. ${ }^{51}$ Accumulating evidence documents that deregulation of apoptosis is identified in inflamed colon and patients with IBD are characterized by higher apoptotic ratio/index in comparison to healthy subjects. ${ }^{52,53}$ Several pro-inflammatory cytokines, including TNF- $\alpha$, are capable of enhancing not only immune response but also apoptosis process. ${ }^{54} \mathrm{An}$ in vitro study conducted by Iablokov et al. found that activation of $\mathrm{PAR}_{2}$ by trypsin, a peptide acting as a $\mathrm{PAR}_{2}$ agonist or a specific agonist is responsible for reduced cleavage of caspases in HT-29 cells pre-treated with cytokines. ${ }^{55}$ Moreover, administration of siRNA against F2RL1 reversed the antiapoptotic effect of $\mathrm{PAR}_{2}$ activation. Iablokov et al. noted that $\mathrm{PAR}_{2}$ may affect apoptosis of colonic epithelial cells acting with mitogen-activated protein kinase kinase, extracellular signal regulated kinase and phosphoinositide 3 kinase/v-Akt (Ak strain transforming) murine thymoma viral oncogene signaling pathways. ${ }^{55}$ In line with the above-mentioned observations, Her et al. documented that $\mathrm{PAR}_{2}$ inhibition enhanced apoptosis related to colitis, which was observed at the protein level in murine model treated with a high-fat diet. ${ }^{56}$ Additionally, the link between apoptosis and autophagy where
$\mathrm{PAR}_{2}$ seems to play a critical role was noted. Autophagy is a process closely associated with cell survival and the connection between apoptosis and autophagy is bidirectional and both processes are overlapping. Experimental evidence found that diet intervention using high-fat diet in mice with $F 2 r l l$ deletion not only aggravated colitis but also affected autophagy-related genes and proteins. ${ }^{56}$ However, association between $\mathrm{PAR}_{2}$, autophagy and colitis is poorly described and further investigations are needed to clarify how $\mathrm{PAR}_{2}$ may affect this process in IBD.

\section{Significance of Protease-Activated Receptors in Thromboembolism Events in Inflammatory Bowel Diseases}

Significance of $\mathrm{PAR}_{1}$ is highlighted not only during immune action mediated by specific immune cells or regulation of colon permeability but also by platelet reactivity in patients with $\mathrm{CD}$. An ex vivo study, whereby multiple electrode aggregometry analysis was conducted by Schmid et al., documented higher reactivity of platelets in response to $\mathrm{PAR}_{1}$ activation by a specific peptide in patients with active stage of $\mathrm{CD}$ in comparison to patients with $\mathrm{CD}$ in remission phase or the control group. ${ }^{57}$ The same pattern of changes upon $\mathrm{PAR}_{1}$ activation was confirmed in an analysis of platelet-monocyte aggregates. Moreover, activation of $\mathrm{PAR}_{1}$ by specific peptide corresponded with enhanced level of P-selectin in platelets obtained from CD patients with inactive and active stage of disease when compared to control group. ${ }^{57}$

In another study it was documented that patients with IBD are characterized by a higher risk of the development of thromboembolism events when compared to general population, especially in patients with active stage of disease. ${ }^{58}$ From the clinical point of view, co-existence of both IBD and thromboembolism events significantly affects mortality. Beyond immune response dysfunctions, which are preliminary hallmarks observed in $\mathrm{UC}$ and $\mathrm{CD}$, patients with IBD are characterized by abnormalities of coagulation factors, thrombin and fibrin generation and formation as well as platelets variations. ${ }^{59-62}$ In fact, in patients with IBD in active stage with a higher number of platelets, their enhanced activity and aggregation potential were documented. ${ }^{61,62}$ This evidence, together with data provided by Schmid et al., suggests that PARs affect the function of platelets and activity modulation of PARs seems to be critical in the prevention of thromboembolism 
events. ${ }^{57}$ Of note, peptide agonists of $\mathrm{PAR}_{4}$ seem to be responsible for platelet activation in active phase of $\mathrm{CD}$, which is manifested by both increased aggregation and increased formation of platelets adhering to monocytes. ${ }^{57}$ Monocytes are not the only type of immune cells which cooperate with platelets. It should also be noted that cathepsin G/PAR 4 axis seems to be responsible for plateletneutrophil interaction at the site of vascular injury and inflammation. ${ }^{63}$ In fact, both receptors, ie $\mathrm{PAR}_{1}$ and $\mathrm{PAR}_{4}$, play an essential role in thromboembolic diseases, which are frequently observed in IBD patients. Nevertheless, additional studies employing in vivo models, not only observational studies, are needed to explore clinical significance of PARs activity modulation in both IBD and thromboembolism events.

\section{Protease-Activated Receptors in the Promotion of the Inflammation- Dysplasia-Carcinoma Sequence}

Finally, PARs significance was highlighted in the inflammation-dysplasia-carcinoma sequence. It has to be mentioned that limited evidence is available about the role of PARs in the neoplastic transformation of the colon in IBD patients. However, colitis is one of the risk factors for colorectal cancer development. In fact, the risk of colorectal cancer for the UC patient is estimated as $2 \%$ after 10 years, $8 \%$ after 20 years and $18 \%$ after 30 years of disease. ${ }^{64} \mathrm{Ke}$ et al. found that mice with F2rll depletion treated with azoxymethane (AOM) and dextran sodium sulfate (DSS) - chemical inducers of neoplastic transformation of the colon - were characterized by more severe colitis, with enhanced expression of pro-inflammatory cytokines and chemokines than wild type mice treated with AOM and DSS. $^{33}$ Additionally, in the colon of $\mathrm{F}_{2 \mathrm{rll}}^{-/-}$mice with colitis-associated colorectal cancer, larger and more differentiated adenocarcinomas were found when compared to wild type animals injected with AOM and treated with DSS, which suggests that $\mathrm{PAR}_{2}$ participates in the progression of neoplastic transformation of the colon associated with colitis. ${ }^{33}$ The mechanism by which $\mathrm{PAR}_{2}$ affects the progression of colitis and colitisassociated colorectal cancer seems to be related to modulation of the microenvironment composition of the colon. In fact, it was proven that the colons of F2rll knockout mice with chemically induced colitis-associated colorectal cancer were characterized by tumor-associated macrophages and myeloid-derived suppressor cell accumulation as well as T cell reduction, leading to immunosuppressive microenvironment development and enhanced production of oxygen species mediated by signal transducer and activator of transcription $3 .^{33}$

\section{Clinical Studies on Agents Targeting PARs}

In 2014, FDA, and one year later EMA, approved the first agent against $\mathrm{PAR}_{1}$, i.e. Vorapaxar, known as a Zontivity or SCH530348 designed for the prevention of thrombotic cardiovascular events. ${ }^{65}$ Vorapaxar inhibits aggregation of platelets induced by thrombin and mediated by $\mathrm{PAR}_{1}$ and is the only agent currently available with a mechanism of action based on $\mathrm{PAR}_{1}$ activity modulation. Another $\mathrm{PAR}_{1}$ antagonist, Atopaxar known as E5555, belongs to small molecule class agents with a mechanism of action similar to Vorapaxar. Nevertheless, according to Phase II clinical trials, Atopaxar usage wascharacterized by significant side effects in patients with acute coronary syndrome and chronic coronary artery disease and did not advance to Phase III. ${ }^{66,67}$ It has to be noted that numerous studies suggest that PARs could be used as a target in the therapy of IBD, but no clinical trials have yet been attempted. According to the ClinicalTrials.gov database, only one ongoing observational study is registered (NCT03011151). As stated by the authors, association between gastrointestinal function, gastric motility, and integrity of epithelial barrier with levels of $\mathrm{PAR}_{2}$ agonist, zonulin and serine protease will be analyzed in pediatric patients with gastrointestinal disorders before surgery and after surgery. On the other hand, along with the abovementioned reports about the link between IBD and thromboembolism events, PARs agents may be a promising tool not only in the prevention of thromboembolism events in IBD patients but also as a regulator of colitis. However, the suggested bidirectional action of PARs has to be evaluated in pre-clinical and clinical studies.

\section{Conclusions}

Taken together, data from recent years provide evidence that PARs are crucial regulators of the progression of IBD. From the clinical point of view, the expression of PARs seems to be an indicator of disease severity. On the other hand, the significance of PARs in disease related to IBD is highlighted. Experimental findings documented that PARs modulate the immune response, which supports several processes involving cytokine and chemokine production/ 
secretion and immune cell infiltration as well as cell-cell interaction. Nevertheless, further studies are needed to determine the therapeutic potential of PARs in patients with $\mathrm{CD}$ and $\mathrm{UC}$.

\section{Acknowledgments}

This work was supported by statutory funds of Department of Cytobiochemistry, Faculty of Biology and Environmental Protection, University of Lodz, as well as Department of Biochemistry (503/1-156-04/503-11-001-19 to J.F.) and Department of Digestive Tract Diseases (503/1-002-01/ 503-11-001-19-00 to E.M.W.), Faculty of Medicine, Medical University of Lodz. D.J. was supported by the Foundation for Polish Science (FNP, START 30.2021).

\section{Disclosure}

The authors report no conflicts of interest in this work.

\section{References}

1. Lu JT, Xu AT, Shen J, Ran ZH. Crosstalk between intestinal epithelial cell and adaptive immune cell in intestinal mucosal immunity. J Gastroenterol Hepatol. 2017;32(5):975-980. doi:10.1111/jgh.13723

2. Xie Y, Chen L, Lv X, et al. The levels of serine proteases in colon tissue interstitial fluid and serum serve as an indicator of colorectal cancer progression. Oncotarget. 2016;7(22):32592-32606. doi:10.18632/oncotarget. 8693

3. Curciarello R, Sobande T, Jones S, et al. Human neutrophil elastase proteolytic activity in ulcerative colitis favors the loss of function of therapeutic monoclonal antibodies. J Inflamm Res. 2020;13:233-243. doi:10.2147/jir.s234710

4. Pedersen G, Saermark T, Kirkegaard T, Brynskov J. Spontaneous and cytokine induced expression and activity of matrix metalloproteinases in human colonic epithelium. Clin Exp Immunol. 2009;155 (2):257-265. doi:10.1111/j.1365-2249.2008.03836.x

5. Meijer MJ, Mieremet-Ooms MA, van der Zon AM, et al. Increased mucosal matrix metalloproteinase-1, $-2,-3$ and -9 activity in patients with inflammatory bowel disease and the relation with Crohn's disease phenotype. Dig Liver Dis. 2007;39(8):733-739. doi:10.1016/j.dld.2007.05.010

6. Rath T, Roderfeld M, Graf J, et al. Enhanced expression of MMP-7 and MMP-13 in inflammatory bowel disease: a precancerous potential? Inflamm Bowel Dis. 2006;12(11):1025-1035. doi:10.1097/01.mib.0000234133.97594.04

7. Yablecovitch D, Kopylov U, Lahat A, et al. Serum MMP-9: a novel biomarker for prediction of clinical relapse in patients with quiescent Crohn's disease, a post hoc analysis. Therap Adv Gastroenterol. 2019;12:1756284819881590. doi:10.1177/1756284819881590

8. Biancheri P, Brezski RJ, Di Sabatino A, et al. Proteolytic cleavage and loss of function of biologic agents that neutralize tumor necrosis factor in the mucosa of patients with inflammatory bowel disease. Gastroenterology. 2015;149(6):1564-1574.e3. doi:10.1053/j. gastro.2015.07.002

9. Ardizzone S, Bianchi Porro G. Biologic therapy for inflammatory bowel disease. Drugs. 2005;65(16):2253-2286. doi:10.2165/ 00003495-200565160-00002

10. Ben-Horin S, Chowers Y. Tailoring anti-TNF therapy in IBD: drug levels and disease activity. Nat Rev Gastroenterol Hepatol. 2014;11 (4):243-255. doi:10.1038/nrgastro.2013.253
11. Guerra I, Bermejo F. Management of inflammatory bowel disease in poor responders to infliximab. Clin Exp Gastroenterol. 2014;7:359-367. doi:10.2147/ceg.s45297

12. Jablaoui A, Kriaa A, Mkaouar H, et al. Fecal serine protease profiling in inflammatory bowel diseases. Front Cell Infect Microbiol. 2020;10:21. doi:10.3389/fcimb.2020.00021

13. Motta JP, Rolland C, Edir A, et al. Epithelial production of elastase is increased in inflammatory bowel disease and causes mucosal inflammation. Mucosal Immunol. 2021;14(3):667-678. doi:10.1038/ s41385-021-00375-w

14. Motta JP, Palese S, Giorgio C, et al. Increased mucosal thrombin is associated with crohn's disease and causes inflammatory damage through protease-activated receptors activation. J Crohns Colitis. 2021;15(5):787-799. doi:10.1093/ecco-jcc/jjaa229

15. Denadai-Souza A, Bonnart C, Tapias NS, et al. Functional proteomic profiling of secreted serine proteases in health and inflammatory bowel disease. Sci Rep. 2018;8(1):7834. doi:10.1038/s41598-01826282-y

16. Galipeau HJ, Caminero A, Verdu EF. Increased bacterial proteolytic activity detected before diagnosis of ulcerative colitis. Inflamm Bowel Dis. 2021;12:e144. doi:10.1093/ibd/izab144

17. Galipeau HJ, Caminero A, Turpin W, et al. Novel fecal biomarkers that precede clinical diagnosis of ulcerative colitis. Gastroenterology. 2021;160(5):1532-1545. doi:10.1053/j.gastro.2020.12.004

18. Vu TK, Hung DT, Wheaton VI, Coughlin SR. Molecular cloning of a functional thrombin receptor reveals a novel proteolytic mechanism of receptor activation. Cell. 1991;64(6):1057-1068. doi:10.1016/ 0092-8674(91)90261-v

19. Gerszten RE, Chen J, Ishii M, et al. Specificity of the thrombin receptor for agonist peptide is defined by its extracellular surface. Nature. 1994;368(6472):648-651. doi:10.1038/368648a0

20. Blackhart BD, Emilsson K, Nguyen D, et al. Ligand cross-reactivity within the protease-activated receptor family. J Biol Chem. 1996;271 (28):16466-16471. doi:10.1074/jbc.271.28.16466

21. O'Brien PJ, Prevost N, Molino M, et al. Thrombin responses in human endothelial cells. Contributions from receptors other than PAR1 include the transactivation of PAR2 by thrombin-cleaved PAR1. J Biol Chem. 2000;275(18):13502-13509. doi:10.1074/ jbc.275.18.13502

22. Paing MM, Stutts AB, Kohout TA, Lefkowitz RJ, Trejo J. Beta Arrestins regulate protease-activated receptor-1 desensitization but not internalization or Down-regulation. J Biol Chem. 2002;277 (2):1292-1300. doi:10.1074/jbc.M109160200

23. Wang P, DeFea KA. Protease-activated receptor-2 simultaneously directs beta-arrestin-1-dependent inhibition and Galphaq-dependent activation of phosphatidylinositol 3-kinase. Biochemistry. 2006;45 (31):9374-9385. doi:10.1021/bi0602617

24. Kaneider NC, Leger AJ, Agarwal A, et al. 'Role reversal' for the receptor PAR1 in sepsis-induced vascular damage. Nat Immunol. 2007;8(12):1303-1312. doi:10.1038/ni1525

25. Jensen DD, Zhao P, Jimenez-Vargas NN, et al. Protein Kinase D and G $\beta \gamma$ Subunits Mediate Agonist-evoked Translocation of Proteaseactivated Receptor-2 from the Golgi Apparatus to the Plasma Membrane. J Biol Chem. 2016;291(21):11285-11299. doi:10.1074/ jbc.M115.710681

26. Mulè F, Pizzuti R, Capparelli A, Vergnolle N. Evidence for the presence of functional protease activated receptor 4 (PAR4) in the rat colon. Gut. 2004;53(2):229-234. doi:10.1136/gut.2003.021899

27. Chin AC, Lee WY, Nusrat A, Vergnolle N, Parkos CA. Neutrophilmediated activation of epithelial protease-activated receptors- 1 and -2 regulates barrier function and transepithelial migration. $J$ Iттииоl. 2008;181(8):5702-5710. doi:10.4049/jimmunol.181.8.5702

28. Dabek M, Ferrier L, Roka R, et al. Luminal cathepsin $g$ and protease-activated receptor 4: a duet involved in alterations of the colonic epithelial barrier in ulcerative colitis. Am J Pathol. 2009;175 (1):207-214. doi:10.2353/ajpath.2009.080986 
29. Saeed MA, Ng GZ, Däbritz J, et al. Protease-activated Receptor 1 Plays a Proinflammatory Role in Colitis by Promoting Th17-related Immunity. Inflamm Bowel Dis. 2017;23(4):593-602. doi:10.1097/ mib.0000000000001045

30. Cenac N, Coelho AM, Nguyen C, et al. Induction of intestinal inflammation in mouse by activation of proteinase-activated receptor-2. Am J Pathol. 2002;161(5):1903-1915. doi:10.1016/ s0002-9440(10)64466-5

31. Maeda S, Ohno K, Uchida K, et al. Intestinal protease-activated receptor-2 and fecal serine protease activity are increased in canine inflammatory bowel disease and may contribute to intestinal cytokine expression. $J$ Vet Med Sci. 2014;76(8):1119-1127. doi:10.1292/jvms.14-0060

32. Kim JA, Choi SC, Yun KJ, et al. Expression of protease-activated receptor 2 in ulcerative colitis. Inflamm Bowel Dis. 2003;9 (4):224-229. doi:10.1097/00054725-200307000-00002

33. Ke Z, Wang C, Wu T, Wang W, Yang Y, Dai Y. PAR2 deficiency enhances myeloid cell-mediated immunosuppression and promotes colitis-associated tumorigenesis. Cancer Lett. 2020;469:437-446. doi:10.1016/j.canlet.2019.11.015

34. Tahara T, Shibata T, Nakamura M, et al. Promoter methylation of protease-activated receptor (PAR2) is associated with severe clinical phenotypes of ulcerative colitis (UC). Clin Exp Med. 2009;9 (2):125-130. doi:10.1007/s10238-008-0025-x

35. Gould NJ, Davidson KL, Nwokolo CU, Arasaradnam RP. A systematic review of the role of DNA methylation on inflammatory genes in ulcerative colitis. Epigenomics. 2016;8(5):667-684. doi:10.2217/epi-2016-0006

36. Fiorucci S, Mencarelli A, Palazzetti B, et al. Proteinase-activated receptor 2 is an anti-inflammatory signal for colonic lamina propria lymphocytes in a mouse model of colitis. Proc Natl Acad Sci U S A. 2001;98(24):13936-13941. doi:10.1073/pnas.241377298

37. Cenac N, Cellars L, Steinhoff $M$, et al. Proteinase-activated receptor-1 is an anti-inflammatory signal for colitis mediated by a type 2 immune response. Inflamm Bowel Dis. 2005;11 (9):792-798. doi:10.1097/01.mib.0000177506.71784.bd

38. Christerson U, Keita AV, Söderholm JD, Gustafson-Svärd C. Increased expression of protease-activated receptor-2 in mucosal mast cells in Crohn's ileitis. J Crohns Colitis. 2009;3(2):100-108. doi:10.1016/j.crohns.2008.11.003

39. Patel M, Shah G. Investigation of Possible Role of the PAR-2 Receptor in Intestinal Inflammation. $J$ Young Pharm. 2010;2 (1):54-58. doi:10.4103/0975-1483.62214

40. Feng BS, Wu YJ, Zeng XH, et al. Bcl2L12 mediates effects of protease-activated receptor-2 on the pathogenesis of Th2-dominated responses of patients with ulcerative colitis. Arch Biochem Biophys. 2018;657:8-14. doi:10.1016/j.abb.2018.09.003

41. Liu B, Yang MQ, Yu TY, et al. Mast Cell Tryptase Promotes Inflammatory Bowel Disease-Induced Intestinal Fibrosis. Inflamm Bowel Dis. 2021;27(2):242-255. doi:10.1093/ibd/izaa125

42. Yen D, Cheung J, Scheerens H, et al. IL-23 is essential for T cell-mediated colitis and promotes inflammation via IL-17 and IL-6. J Clin Invest. 2006;116(5):1310-1316. doi:10.1172/jci21404

43. Mari B, Guerin S, Far DF, et al. Thrombin and trypsin-induced $\mathrm{Ca}(2$ + ) mobilization in human $\mathrm{T}$ cell lines through interaction with different protease-activated receptors. FASEB j. 1996;10(2):309-316. doi:10.1096/fasebj.10.2.8641564

44. Christerson U, Keita AV, Söderholm JD, Gustafson-Svärd C. Potential role of protease-activated receptor-2-stimulated activation of cytosolic phospholipase A(2) in intestinal myofibroblast proliferation: implications for stricture formation in Crohn's disease. J Crohns Colitis. 2009;3(1):15-24. doi:10.1016/j.crohns.2008.10.002

45. Wang F, Graham WV, Wang Y, Witkowski ED, Schwarz BT, Turner JR. Interferon-gamma and tumor necrosis factor-alpha synergize to induce intestinal epithelial barrier dysfunction by up-regulating myosin light chain kinase expression. Am J Pathol. 2005;166(2):409-419. doi:10.1016/s0002-9440(10)62264-х
46. Graham WV, Wang F, Clayburgh DR, et al. Tumor necrosis factor-induced long myosin light chain kinase transcription is regulated by differentiation-dependent signaling events. Characterization of the human long myosin light chain kinase promoter. $J$ Biol Chem. 2006;281(36):26205-26215. doi:10.1074/jbc.M602164200

47. Blair SA, Kane SV, Clayburgh DR, Turner JR. Epithelial myosin light chain kinase expression and activity are upregulated in inflammatory bowel disease. Lab Invest. 2006;86(2):191-201. doi:10.1038/ labinvest. 3700373

48. Gecse K, Róka R, Ferrier L, et al. Increased faecal serine protease activity in diarrhoeic IBS patients: a colonic lumenal factor impairing colonic permeability and sensitivity. Gut. 2008;57(5):591-599. doi:10.1136/gut.2007.140210

49. Róka R, Rosztóczy A, Leveque M, et al. A pilot study of fecal serine-protease activity: a pathophysiologic factor in diarrhea-predominant irritable bowel syndrome. Clin Gastroenterol Hepatol. 2007;5(5):550-555. doi:10.1016/j.cgh.2006.12.004

50. Dabek M, Ferrier L, Annahazi A, et al. Intracolonic infusion of fecal supernatants from ulcerative colitis patients triggers altered permeability and inflammation in mice: role of cathepsin $G$ and protease-activated receptor-4. Inflamm Bowel Dis. 2011;17 (6):1409-1414. doi:10.1002/ibd.21454

51. Sébert M, Denadai-Souza A, Quaranta M, et al. Thrombin modifies growth, proliferation and apoptosis of human colon organoids: a protease-activated receptor 1 - and protease-activated receptor 4-dependent mechanism. $\mathrm{Br} \quad J \quad$ Pharmacol. 2018;175 (18):3656-3668. doi:10.1111/bph.14430

52. Zeissig S, Bojarski C, Buergel N, et al. Downregulation of epithelial apoptosis and barrier repair in active Crohn's disease by tumour necrosis factor alpha antibody treatment. Gut. 2004;53 (9):1295-1302. doi:10.1136/gut.2003.036632

53. Dirisina R, Katzman RB, Goretsky T, et al. p53 and PUMA independently regulate apoptosis of intestinal epithelial cells in patients and mice with colitis. Gastroenterology. 2011;141(3):1036-1045. doi:10.1053/j.gastro.2011.05.032

54. Kim JJ, Lee SB, Park JK, Yoo YD. TNF-alpha-induced ROS production triggering apoptosis is directly linked to Romol and Bcl-X(L). Cell Death Differ. 2010;17(9):1420-1434. doi:10.1038/ cdd.2010.19

55. Iablokov V, Hirota CL, Peplowski MA, et al. Proteinase-activated receptor 2 (PAR2) decreases apoptosis in colonic epithelial cells. $J$ Biol Chem. 2014;289(49):34366-34377. doi:10.1074/jbc.M114.610485

56. Her JY, Lee Y, Kim SJ, et al. Blockage of protease-activated receptor 2 exacerbates inflammation in high-fat environment partly through autophagy inhibition. Am J Physiol Gastrointest Liver Physiol. 2021;320(1):G30-g42. doi:10.1152/ajpgi.00203.2020

57. Schmid W, Vogelsang H, Papay P, et al. Increased responsiveness to thrombin through protease-activated receptors (PAR)- 1 and -4 in active Crohn's disease. J Crohns Colitis. 2014;8(6):495-503. doi:10.1016/j.crohns.2013.11.001

58. Grainge MJ, West J, Card TR. Venous thromboembolism during active disease and remission in inflammatory bowel disease: a cohort study. Lancet. 2010;375(9715):657-663. doi:10.1016/ s0140-6736(09)61963-2

59. Conlan MG, Haire WD, Burnett DA. Prothrombotic abnormalities in inflammatory bowel disease. Dig Dis Sci. 1989;34(7):1089-1093. doi: $10.1007 / \mathrm{bf} 01536380$

60. Hudson M, Hutton RA, Wakefield AJ, Sawyerr AM, Pounder RE. Evidence for activation of coagulation in Crohn's disease. Blood Coagul Fibrinolysis. 1992;3(6):773-778. doi:10.1097/00001721199212000-00011

61. Collins CE, Cahill MR, Newland AC, Rampton DS. Platelets circulate in an activated state in inflammatory bowel disease. Gastroenterology. 1994;106(4):840-845. doi:10.1016/0016-5085(94) 90741-2 
62. Danese S, Katz JA, Saibeni S, et al. Activated platelets are the source of elevated levels of soluble CD40 ligand in the circulation of inflammatory bowel disease patients. Gut. 2003;52(10):1435-1441. doi:10.1136/gut.52.10.1435

63. Sambrano GR, Huang W, Faruqi T, Mahrus S, Craik C, Coughlin SR. Cathepsin G activates protease-activated receptor-4 in human platelets. J Biol Chem. 2000;275(10):6819-6823. doi:10.1074/ jbc.275.10.6819

64. Eaden JA, Abrams KR, Mayberry JF. The risk of colorectal cancer in ulcerative colitis: a meta-analysis. Gut. 2001;48(4):526-535. doi:10.1136/gut.48.4.526

65. Fala L. Zontivity (Vorapaxar), First-in-Class PAR-1 Antagonist, Receives FDA Approval for Risk Reduction of Heart Attack, Stroke, and Cardiovascular Death. Am Health Drug Benefits. 2015;8 (SpecFeature):148-151.
66. O’Donoghue ML, Bhatt DL, Wiviott SD, et al. Safety and tolerability of atopaxar in the treatment of patients with acute coronary syndromes: the lessons from antagonizing the cellular effects of Thrombin-Acute Coronary Syndromes Trial. Circulation. 2011;123 (17):1843-1853. doi:10.1161/circulationaha.110.000786

67. Goto S, Ogawa H, Takeuchi M, Flather MD, Bhatt DL. Double-blind, placebo-controlled Phase II studies of the protease-activated receptor 1 antagonist E5555 (atopaxar) in Japanese patients with acute coronary syndrome or high-risk coronary artery disease. Eur Heart J. 2010;31(21):2601-2613. doi:10.1093/eurheartj/ehq320

\section{Publish your work in this journal}

The Journal of Inflammation Research is an international, peerreviewed open-access journal that welcomes laboratory and clinical findings on the molecular basis, cell biology and pharmacology of inflammation including original research, reviews, symposium reports, hypothesis formation and commentaries on: acute/chronic inflammation; mediators of inflammation; cellular processes; molecular mechanisms; pharmacology and novel anti-inflammatory drugs; clinical conditions involving inflammation. The manuscript management system is completely online and includes a very quick and fair peerreview system. Visit http://www.dovepress.com/testimonials.php to read real quotes from published authors.

Submit your manuscript here: https://www.dovepress.com/journal-of-inflammation-research-journal 